\title{
Agent Based Model of Livestock Movements
}

\author{
D.J. Miron \\ CSIRO Livestock Industries, Australia \\ david.miron@csiro.au
}

,I. V. Emelyanova

CSIRO Livestock Industries, Australia irina.emelyanova@csiro.au

,G. E. Donald

CSIRO Livestock Industries, Australia graham.donald@csiro.au

,G. M. Garner

Department Agriculture Fisheres and Forestry, Australia graeme.garner@affa.gov.au

\begin{abstract}
The modelling of livestock movements within Australia is of national importance for the purposes of the management and control of exotic disease spread, infrastructure development and the economic forecasting of livestock markets. In this paper an agent based model for the forecasting of livestock movements is presented. This models livestock movements from farm to farm through a saleyard. The decision of farmers to sell or buy cattle is often complex and involves many factors such as climate forecast, commodity prices, the type of farm enterprise, the number of animals available and associated off-shore effects. In this model the farm agent's intelligence is implemented using a fuzzy decision tree that utilises two of these factors. These two factors are the livestock price fetched at the last sale and the number of stock on the farm. On each iteration of the model farms choose either to buy, sell or abstain from the market thus creating an artificial supply and demand. The buyers and sellers then congregate at the saleyard where livestock are auctioned using a second price sealed bid. The price time series output by the model exhibits properties similar to those found in real livestock markets.
\end{abstract}




\section{Introduction}

The development of a national scale spatial simulation model for the modelling of livestock movements has been identified as a project of national importance by the Australian Department of Agriculture Fisheries and Forestry. Such a model would have applications in the development of policy and risk assessments surrounding exotic disease outbreaks, economic forecasting and infrastructure planning.

In this work an agent based modelling (ABM) approach is utilised for this purpose. The ABM approach is grounded in complex systems science and can be used to model non-linear systems such as those found in social and biological systems. For example, ABM techniques have been used in the development of models for the study of financial markets $[1,2,11]$.

To utilise an ABM approach requires the identification of the agent types that are participating within the complex system and the development of the intelligence within the agents. The development of the intelligence within the agents is a key factor in determining realistic outputs for the model and is an area of active research [12].

The modelling of livestock movements can be achieved through the modelling of livestock markets as it is the latter that drives the former. Livestock markets are complex systems influenced by many factors. For example, rainfall, livestock prices, interest rates and feed prices all affect the decision making of stakeholders within the system which in turn influences the market.

For the purposes of this work the initial focus has been on beef cattle markets. Within the beef cattle sector the stakeholders are farmers, abattoirs, exporters, traders and feedlots. It is these stakeholders that are the agents within the model. However, in an effort to gauge the effectiveness of the ABM approach the model was simplified by considering only the farmer and saleyard agents. This simplification meant that only farm to farm movements through a saleyard were modelled.

Using this simplification and by implementing simple intelligence with farmer agents and a saleyard auction, it is shown that the price time series generated by the model has the same statistical and chaotic properties as those found in actual cattle markets.

This paper is divided into four sections. In $\S 2$ the livestock market is discussed and the models agents identified. In $\S 3$ the ABM that was implemented is described while in $\S 4$ results are presented. Conclusions and further work are given in $\S 5$.

\section{The Market}

A single trade between a buyer and a seller does not constitute a market. A market is created through a collection of trades between multiple buyers and sellers for a common commodity. The existence of a market is demonstrated through prices and trade volumes. The market operates under a number of 
different sets of rules such as the rules of supply and demand and the rules of trading.

Within cattle markets agents can buy or sell or as in the case of farmers take on the dual role of buyer and seller. The agents and their interactions are illustrated in Fig. 1.

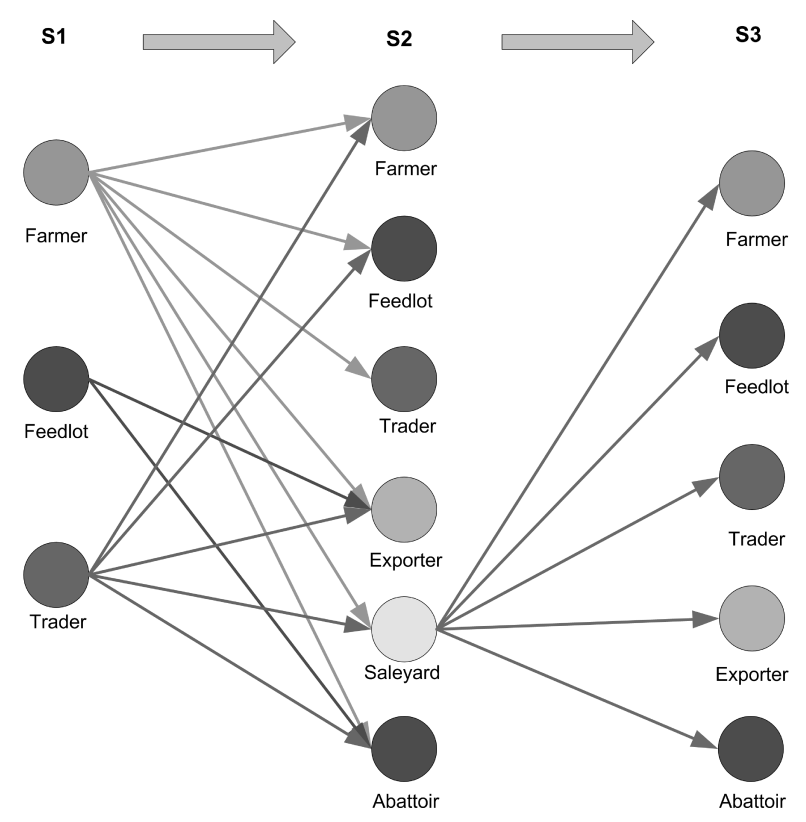

Figure 1: The daily trade cycle.

Fig. 1 illustrates the daily trade cycle of the agents participating in the cattle market. Farmers are the primary producers of livestock and depending upon their enterprise type may breed or fatten cattle for the purposes of sale. Traders are opportunists within the market only buying livestock to sell again in the short term. Exporters buy livestock for the purposes of export while abattoirs buy livestock for the purposes of slaughter. Feedlots are a special instance of a farmer in that they buy cattle for fattening before selling them on to an abattoir or an exporter. The saleyard has also been identified as an agent as it is a place where buyers and sellers congregate for the purposes of trading livestock through an auction.

Further, the daily trade cycle given in Fig. 1 is broken up into three stages, S1, S2 and S3. Stage S1 is made up of the agents that offer their cattle for sale while stage $\mathrm{S} 2$ is the group of agents, with the exception of saleyards, that would buy cattle. Again taking the example of farmers, they have the choice of selling directly to other agents such as other farmers and feedlots or offering their cattle for sale through a saleyard. It is the saleyard that necessitates the need for stage S3. Stage S3 is made up of agents that purchase cattle through a saleyard. 
Immediately evident is the magnification role, in terms of the distribution of animals, that saleyards have within cattle markets.

\section{The Agent Based Model}

To reduce the complexity of the cattle market an agent based model was implemented that focused on farm to farm trades through a saleyard. This necessitated the development of a farmer agent's intelligence for decision making for the purposes of creating an artificial supply and demand. This decision making by farmers was centered around two questions: "Should I buy/sell cattle?" and if so, "How many cattle should I buy/sell?".

There are many factors that influence farmer decision making. Some of these factors are on farm cattle numbers, rainfall, commodity price, the region in which their farm resides, the types of animals that the farm produces and the type of farm enterprise.

In the development of the model, two of these factors were implemented. These two factors were the on farm cattle numbers (stock on hand) $s$ and the market price $p$. For example, when price is low and stock on hand is low then the farmers decision would be to buy and not to sell. Conversely, if the price $p$ is high and the stock on hand $s$ is high then the farmers decision would be to sell and not buy. These factors and their consequent decisions can be represented by way of a matrix as depicted in Fig. 2:
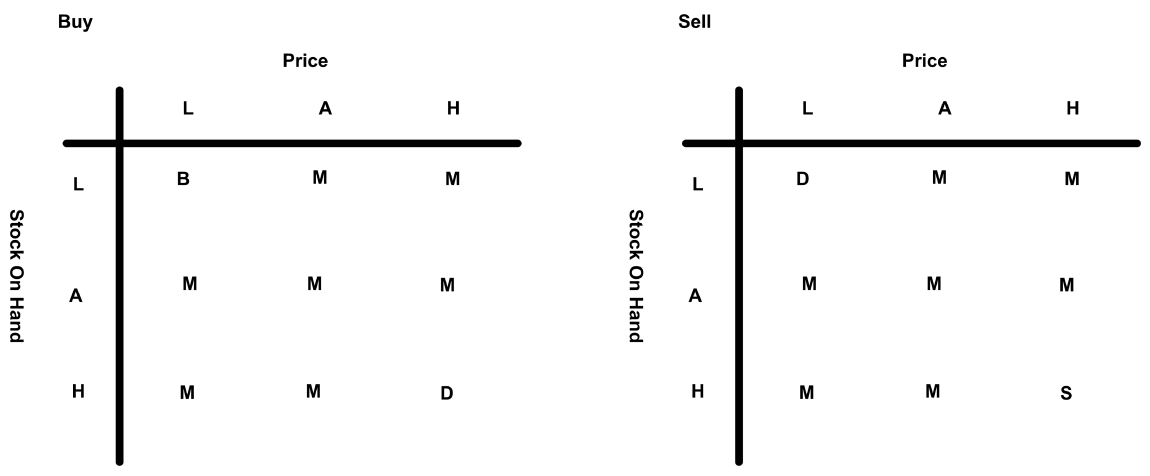

Figure 2: The sell/buy decision matrices.

The entries in the two matrices represent the cases don't sell/buy (D), buy (B), sell (S) and maybe buy/sell (M). The columns/rows of the matrix depict the status of each of the two factors while the values of the factors can either be low $(\mathrm{L})$, average $(\mathrm{A})$ or high $(\mathrm{H})$.

These values low $(\mathrm{L})$, average $(\mathrm{A})$ or high $(\mathrm{H})$ were represented by fuzzy variables. Fuzzy variables were first introduced by Zadeh [13]. The fuzzy variables in the decision matrices shown in Fig. 2 low, average and high were defined as: 


$$
\mu=\left\{\begin{array}{l}
\text { low }=0 \geq \text { price } \leq 0.9 \\
\text { average }=\text { price } \in \psi(1.0,0.1) \\
\text { high }=1.1 \geq \text { price } \leq 2.0
\end{array}\right.
$$

Within equation 1 the function $\psi$ is a trapezoidal function centered at 1.0 with endpoints at 0.9 and 1.1 .

For the purposes of modelling, a saleyard in South East Queensland was chosen. The farms that participated in the market were those farms whose closest selling centre was the chosen saleyard. The stock on hand $s$ for a farm $i$ was calculated using the ratio of actual stock on hand $\hat{s}_{i}$ by average stock on hand $\bar{s}_{i}$. The value for the average stock on hand $\bar{s}_{i}$ was derived from Ag-Stats census data [3]. Thus, as a farm $i$ bought and sold stock the fuzzy value of the stock on hand $\mu_{i}^{s}$ would change.

Initially, the current price $\rho$ at the saleyard was set to $\rho=1.0$ which yielded a fuzzy value $\mu^{\rho}=$ average. The saleyard's current price $\rho$ then fluctuated as a result of an auction process.

On each time step $t$ farms were selected to buy and/or sell based on the outcome of applying the fuzzy decision matrices in Fig. 2. However, the decision matrices of Fig. 2 were modified to include a graded multiplication factor. The resulting matrices are given in Fig. 3.
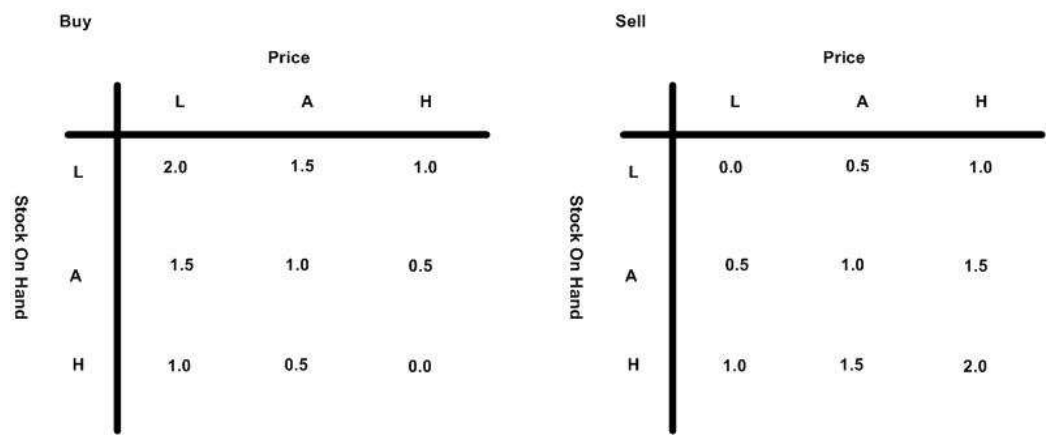

Figure 3: The multipliers for selection pressures of buying and selling.

The multiplication factors were used to compute a selection pressure for each farm $i$ for each of the cases of buying and selling. The selection pressure for a selling farm $\gamma_{i}^{\text {sell }}$ was calculated by first calculating the probability that farm $i$ would sell. This probability $p_{i}$ (sell) was the expected number of selling farms divided by the total number of farms. The expected number of selling farms was determined through expert estimates gathered by the Australian Department of Fisheries and Forestry (DAFF) [4].

The multiplier for farm $i$ to sell $m_{i}^{\text {sell }}$ was then applied to the probability of a farm selling $p_{i}($ sell $)$ yielding:

$$
\gamma_{i}^{\text {sell }}=m_{i}^{\text {sell }} \times p_{i}(\text { sell }) .
$$


A random number $r_{i}$ where $r_{i} \in[0,1)$ for farm $i$ was then computed and applied to the function $g\left(r_{i}\right)$ where:

$$
g\left(r_{i}\right)= \begin{cases}\text { participate } & \text { if } r_{i}<\gamma_{i}^{\text {sell }} \\ \text { abstain } & \text { otherwise }\end{cases}
$$

If farm $i$ was found to be a selling farm participating in the market then the number of stock needed to be calculated for sending to market. This was the actual stock on hand $0.1 \hat{s} r_{j}$ where $r_{j}$ was a random number and $r_{j} \in[0,1)$.

The farms selection pressure for buying $\gamma_{i}^{\text {buy }}$ was calculated in a similar manner and the decision made to buy using the same function $g\left(r_{i}\right)$. The number of stock to buy was also computed using the formula $0.1 \hat{s} r_{j}$ where $r_{j}$ is a random number and $r_{j} \in[0,1)$.

Having completed the decision making process for buying and selling, the farms participating in the market began to buy and sell at auction. The actual auction technique implemented was a second price sealed bid. That is each buyer made one bid on the current lot and the bid was secret. That is no bidder knew what other bidders had bid. The winning bidder was the bidder with the highest bid but they only paid the losing bidder's price.

The second price sealed bid auction was used as it is weakly equivalent to an English auction [10]. In an English auction bidding for a good starts at low price and ascends until there is only one bidder left. The last bidder left then pays the price for the good that they bid. Further, in an English auction bids are public. In Australian saleyards a variant of the English auction is used. In this variant the price paid is calculated as follows. If the bids are progressing in 1 cent increments per kilogram then the price paid is the highest bid less $80 \%$ of the bid increment. Likewise, if bidding is progressing in 0.5 cent increments per kilogram then the pice paid per kilogram is the highest bid less $80 \%$ of the bid increment. For example, if the highest price was 150 cents then the winning bidder would pay 149.2 cents. If the increment was half cent bids then the price would be 149.6 cents.

Within the model, bidders bid on each lot until their required number of stock is purchased. After each round of bidding a market supply and demand factor was calculated. This modified the starting price of the bids for the lot. Actual bids were then modified by a choosing a random number from a Gaussian distribution centered at the "last lot price" with a variance of 0.005 .

\section{Results}

A random output of the price time series from the model was taken. This time series was over a period of 1000 time steps. A time step could be considered a weekly sale. This time series was then tested for chaotic behaviour and compared against known cattle market chaos analysis to show that the cattle market created by the model has similar properties to a real cattle market. 
The first step in the analysis of the model time series was to denoise the original signal. The wavelet shrinkage method of Donoho [5] was applied for this purpose. A shrinking threshold of 0.1 was selected for the signal denoising. Fig. 4 shows both the original signal (time series) on the left and the denoised signal on the right.
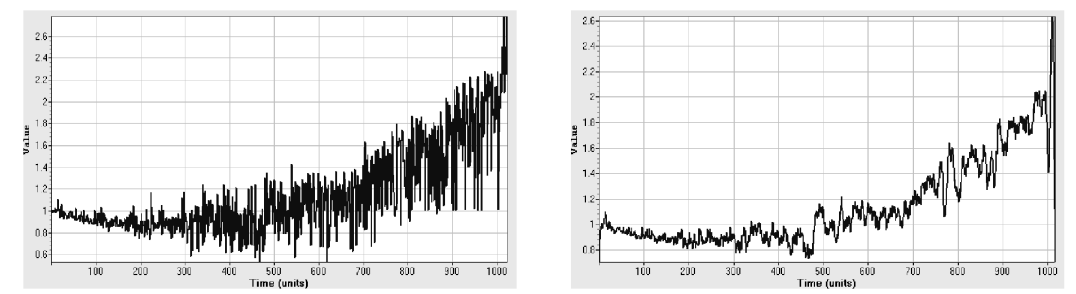

Figure 4: Time series of the original signal (left) and the denoised signal (right).

Fig. 5 shows the phase portraits for the time series of the original signal on the left and the denoised signal on the right.
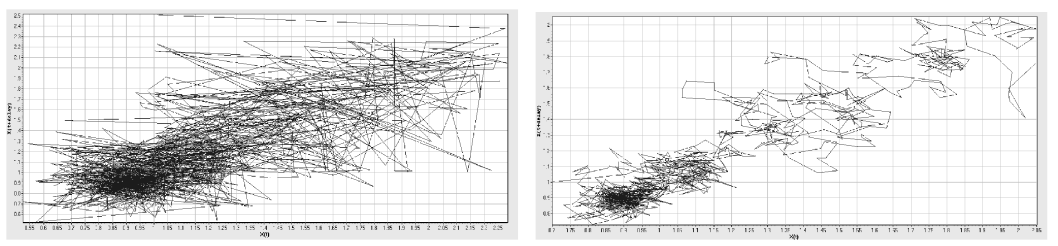

Figure 5: Phase portrait of the original signal (left) and the denoised signal (right).

From Fig. 5 it is evident that there are some similarities in distribution and density of trajectories in both two-dimensional phase space reconstructions. However, the trajectories of the denoised signal are smoother and free of random fluctuations. It may be seen that trajectories are quite mixed, change direction very quickly and build a complicated pattern. Thus, an assumption was made about chaotic behaviour of the model data. All the figures were produced using a freely available software package "Visual Recurrence Analysis 4.9" developed for topological analysis and qualitative assessment of time series [9].

The self-similarity of the model signal was tested by calculating correlation dimension introduced by Grassberger and Procaccia [6]. This measure helps quantify fractal features of the attractors. The graph of correlation dimensions for the reconstructed attractors of the original and denoised signals for the range of embedding dimensions $m=1-17$ and time delay $\tau=11$ is shown in Fig. 6 .

From Fig. 6 (left) it can be seen that the curve for the original signal achieves its plateau at the value $D_{o} 2.54$. The value of the correlation dimension calculated for the denoised signal shown in Fig. 6 (right) was $D_{d} 1.96$ which is lower due to the reduction in the noise as compared to the original signal. This confirms the assumption about chaotic behaviour of the model data as attractors 

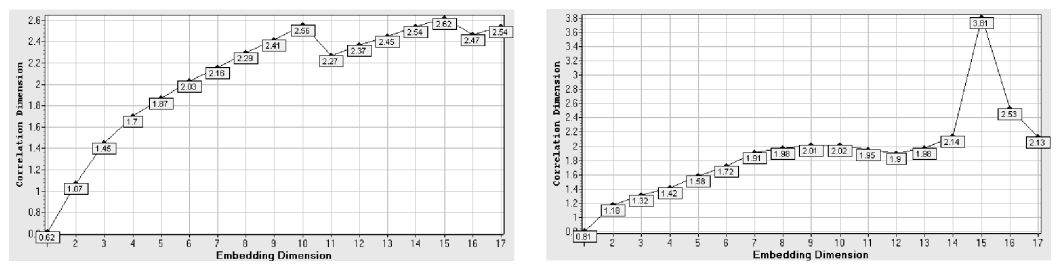

Figure 6: Correlation dimension for the original signal (left) and the denoised signal (right).

with fractional dimensions are typical of chaotic systems. Similar values for the correlation dimension were obtained in an analysis of a real cattle market by Kohzadi and Boyd [8]. This indicates that the behaviour of the model has produced chaotic properties similar to that of actual saleyards.

The fractal features of the model time series were also tested by the calculation of the Hurst exponent $H$ [7]. It is known that a Hurst exponent of $H=1$ results for smooth time series while a Hurst exponent of $H=0.5$ characterises noise and a Hurst exponent of $0.5<=H<=1$ indicates a natural process. A Hurst exponent calculated for the denoised signal was found to have a value of $H=0.62$ while the value of the Hurst exponent estimated for a real cattle market data was found to be a little lower at $H=0.51$ [8].

To continue the analysis of the model time series a recurrence plot of the denoised signal was constructed. The recurrence plot indicated phase transitions and instationarities within the signal. Also, positive values of maximal Lyapunov exponents $L^{l}=0.3$ and $L^{d}=0.6$ calculated for the original and denoised signals respectively indicated the sensitive dependence on initial conditions. This suggests exponential divergence of nearby trajectories of the attractor and chaos.

\section{Conclusions and Further Work}

In this paper an agent based model has been put forward for the modelling of cattle movements. This model focussed on the movement of cattle from farm to farm through a saleyard. Using fuzzy decision tree for the decision making within the farm agents and an auction simulation it was shown that the model produced price time series that had characteristics similar to that of real saleyards. This has laid a strong foundation for further development of the model. It is envisaged that improving the agent decision making processes will be a key factor in improving the model outputs and is an area of active research.

\section{Bibliography}

[1] Arthur, W., "On learning and adaptation in the economy", Tech. Rep. no. 92-07-038, Santa fe Institute, (1992). 
[2] Arthur, W., J. Holland, B. Le Baron, R. Palmer, and P. Tayler, "Asset pricing under endogenous expectations in an artificial stock market" (1996).

[3] "Australian bureau of statistics: Agstats census data" (2001).

[4] Beckett, S., "Saleyard characteristics ausbeef region 6" (2006).

[5] Donoho, D., "De-noising by soft-thresholding", IEEE Trans. Inf. Theory 41 (1994), 613-627.

[6] Grassberger, P., and I. Procaccia, "Measuring the strangeness of strange attaractors", Physica 9D (1983), 30-31.

[7] Hurst, H., "Long-term storage capacity of reservoirs", Transactions of American Society of Civil Engineering 116 (1951), 770-799.

[8] Kohzadi, N., and M. Boyd, "Testing for chaos and nonlinear dynamics in cattle prices", Canadian Journal of Agricultural Economics 43 (1995), 475-484.

[9] Kononov, E., "Visual recurrence analaysis", http://www.myjavaserver.com/ nonlinear/vra/download.html.

[10] Krishna, V., Auction Theory, Academic Press (2002).

[11] LeBaron, B., W. Arthur, and R. Palmer, "Time series properties of an artificial stock market", Journal of Economic Dynamics \& Control 23 (1999), 1487-1516.

[12] Perez, P., Complex Science for a Complex World: Exploring Human Ecosystems with Agents, ANU Epress, Australia, (2006), ch. Chapter 3: Agents, Icons and Idols, pp. 27-56.

[13] ZaDeH, L., "Fuzzy sets", Information and Control 8 (1965), 338-353. 